\title{
Prognostic factors of delayed cerebral ischemia after subarachnoid hemorrhage including CT perfusion: a prospective cohort study
}

\author{
Mohamed Abdel-Tawab ${ }^{1 *}$, Afaf A. Hasan ${ }^{1}$, Mohamed A. Ahmed ${ }^{2}$, Hany M. A. Seif ${ }^{1}$ and Hazem A. Yousif ${ }^{1}$
}

\begin{abstract}
Background: Delayed cerebral ischemia (DCI) is the worst sequel following subarachnoid hemorrhage (SAH), representing a challenge in prediction and prevention. The current study aims to identify the optimum predictors of DCl including CT perfusion (CTP) and to determine the best prognostic thresholds.

This prospective study included 49 SAH patients. All patients were treated with the standard therapy and underwent non-contrast CT, CTP, and CTA within 3 days after SAH. Hunt and Hess and Fisher scales were assessed besides quantitative CTP parameters. The primary endpoint was DCI within 21 days after SAH, defined as clinical deterioration or infarction.
\end{abstract}

Results: Out of 49 eligible patients with SAH, 9 patients developed DCl. Univariate analysis revealed that Hunt and Hess scale, Fisher scale, the presence of a cerebral aneurysm, and mean transit time (MTT) were predictive for DCl. Diagnostic threshold values by ROC curve analysis with optimal sensitivity and specificity were Hunt and Hess scale > 2, Fisher scale $>2$, and MTT of $4.65 \mathrm{~s}$.

Conclusion: MTT is a sensitive and specific predictor of DCl. However, Hunt and Hess scale has the optimal sensitivity and specificity to distinguish between patients who developed DCI and clinically stable patients.

Keywords: CT perfusion, Delayed cerebral ischemia, Fisher scale, Hunt and Hess scale, Subarachnoid hemorrhage, Vasospasm

\section{Background}

Subarachnoid hemorrhage (SAH) is a critical condition with an incidence ranging from 6 to 15 cases per 100, 000 [1]. Following SAH, nearly one-third of patients will die, one-third will survive with a disability, and one-third will survive with good recovery [2]. Besides the direct effect of hemorrhage, rebleeding and DCI are considered the major complications of SAH [3]. The risk of rebleeding has been significantly reduced by early aneurysmal closure [4]. In contrast, DCI is still a significant cause of

\footnotetext{
* Correspondence: dr.m.tawab@gmail.com

${ }^{1}$ Department of Radiology, Faculty of Medicine, Assiut University, Assiut, Egypt

Full list of author information is available at the end of the article
}

death and disability among patients with SAH [5]. DCI occurs mostly due to vasospasm, however, recent studies proved that vasospasm is not considered the only leading factor of DCI as impaired cerebral autoregulation also plays a role [6]. DCI is defined clinically as a new unexplained neurological deficit, (e.g., hemiparesis or aphasia) or a drop in the level of consciousness (more than 2 points on Glasgow Coma Scale) lasting for $2 \mathrm{~h}$ [7]. Radiologically, it is defined as a hypodense area on CT or hyperintense area in FLAIR and DWI MRI [8].

Early diagnosis and aggressive treatment with intraarterial vasodilators and balloon angioplasty must be performed as early as possible to prevent the morbid and fatal consequences of DCI [9]. However, clinical 
diagnosis of DCI in patients with altered consciousness or those who are sedated is impossible as clinical recognition of the drop of consciousness level may not be consistent [10]. Besides, clinical monitoring in fully conscious patients only allows the diagnosis but not the prognosis of $\mathrm{DCI}$, thus limiting initiation of prompt prophylactic therapy [11].

Many parameters have been widely investigated for prediction of DCI, including patient demographics (e.g., age and gender), clinical scales at presentation (e.g., Hunt and Hess scale), the amount of SAH (e.g., Fisher scale), and site of an aneurysm [12].

Among different imaging techniques evaluating cerebral perfusion, CTP is characterized by availability, low costs, rapid imaging, and high spatial resolution [13]. CTP is done by monitoring the first pass of an iodinated contrast agent bolus through the cerebral circulation [14]. A series of slices are produced by repeatedly scanning the same region with a non-moving table, i.e., the same $z$-position [15]. As there is a linear relationship between the iodine concentration and the density changes in the tissue, perfusion parameters are calculated by the deconvolution analysis [14].

Diffusion-weighted imaging (DWI) is the modality of choice in the diagnosis of infarction [16]; however, it only detects the irreversible infarcted tissue which could not replace perfusion studies that are superior in detecting reversibly ischemic tissues [17].

In our study, we aim to investigate the previously mentioned predictors and stress on the role of early CTP including CBF and MTT and to establish prognostic thresholds for DCI.

\section{Methods}

\section{Study participants}

This study was a clinically registered (NCT03214705), prospective cohort study. It included patients with SAH who were admitted to the neurology department from March 2016 to February 2018. The study was approved from the institutional review board and written informed consent was obtained; either from the patients themselves (29 patients) or their relatives (20 patients) as those patients were unconscious or complaining severe headache. All patients received standard treatment including oral nimodipine. The diagnosis of SAH and the site of the origin were determined by CT and CTA. CTP was performed during the first $72 \mathrm{~h}$. Follow-up was done by daily clinical examination to detect any new neurological deficit. Radiological DCI was determined by CT or MRI at the time of deterioration otherwise follow up CT/MRI was performed before discharge at 14th-21st day after ictus. Patients were then classified into two groups: those who developed DCI and those who did not.

\section{Imaging technique}

CT, CTP, and CTA imaging were carried out on a 64channel Multichannel CT scanner (Aquilion; Toshiba Medical Systems, Tokyo, Japan). Non-ionic contrast material (Iopromide, Ultravist, 370; Schering, Berlin, Germany) was used for CTP and CTA.

After non-contrast brain CT was obtained, CTP was performed on 4 adjacent 8 -mm-thick sections, at the level of the basal ganglia. At this level, ACA, MCA, and PCA vascular territories can be visualized. Time delay of $5 \mathrm{~s}$ was achieved after injection of $50 \mathrm{~mL}$ of the nonionic contrast agent at a flow rate of $5 \mathrm{~mL} / \mathrm{s}$, a 45 -s cine series was obtained with the following parameters: 120 $\mathrm{kVp}, 200 \mathrm{~mA}, 0.4 \mathrm{~s}$ rotation time.

CTA was acquired 5 min after the CTP and after injection of $50 \mathrm{~mL}$ saline to wash the brain from residual contrast. Administration of $50 \mathrm{~mL}$ of contrast was achieved at a flow rate of $5 \mathrm{~mL} / \mathrm{s}$. Bolus tracking technique was used to optimize the timing of imaging. Parameters for CTA were $120 \mathrm{kVp}, 350 \mathrm{mAs}$, spiral mode, $0.6 \mathrm{~s}$ rotation time; collimation, $64 \times 0.5 \mathrm{~mm}$; pitch, 0.828 ; section thickness, $0.5 \mathrm{~mm}$; reconstruction interval, $0.4 \mathrm{~mm}$.

\section{Data processing and analysis}

Post-processing of the perfusion maps was performed by Vitrea workstation (Vital Images, Inc.), a commercially available software using the deconvolution method. After defining arterial input and venous output reference points, perfusion maps of cerebral blood volume (CBV), cerebral blood flow (CBF), mean transit time (MTT), and time to peak (TTP) were generated. These maps are assessed both qualitatively and quantitatively by two radiologists and measurements at the areas of least CBF were recorded.

CTA interpretation was focusing on detecting cerebral aneurysms and vasospasm. For aneurysm detection, raw data, MIP, and volume rendering images were used. For vasospasm evaluation, MIP and volume rendering images were used. CTA criteria for vasospasm were determined by arterial narrowing compared with the normal patent vessel diameter. The review of the CTA typically lasted 10-15 min for each patient and was performed by 2 readers in agreement.

\section{Statistical analysis}

For statistical analysis, we used a commercially available software package SPSS release 23.0 program (SPSS for Windows; SPSS Inc, Chicago, IL, USA) and MedCalc version 18.2.1 ((MedCalc Software, 9030 Mariakerke, Belgium).

Continuous data were reported as mean \pm standard deviation if normally distributed and in median (interquartile range) if not normally distributed. Categorical 
data were reported in frequencies and percentages and analyzed with chi-square test when appropriate. Quantitative data for all patients were evaluated for statistically significant differences between both groups utilizing a two-tailed, independent Student's $t$ test if data were normally distributed and Mann-Whitney test if data not normally distributed. Statistical significance was accepted at $p<0.05$. A univariate analysis yielding odds ratios with $95 \%$ confidence intervals $(95 \%$ CIs) was applied to test predictive factors for DCI. Predictors with $p$ $<0.05$ were entered in a ROC curve analysis to detect cut-off values, sensitivity, specificity, positive and negative predictive values, and accuracy.

\section{Results}

A total of 49 patients (22 women) aged 20 to 70 years (mean, 49 years) presented with SAH were included in the statistical analysis of the 64 enrolled patients. Fifteen patients were excluded as the following: 2 patients were excluded due to severe motion artifact, 4 patients were excluded due to improper technique, 3 patients were lost to follow-up, and 6 patients were excluded due to death not attributed to DCI (either not performing follow up CT before death in 2 patients or death from other causes, i.e., rebleeding), shown in Fig. 1.

The characteristics of DCI and non-DCI groups are shown in Table 1. Both groups were homogenous in baseline socio-demographic data without significant differences in mean age, gender and risk factors including hypertension and diabetes. As regards Hunt and Hess scale and Fisher scale at presentation, there were highly significant differences between the two groups ( $p$ value $=0.000,0.014$, respectively). No significant difference was found as regards to the presence of intracerebral hematoma $(p$ value $=0.07)$. The site of SAH showed a significant difference $(p$ value $=0.03)$. Both the presence and site of a cerebral aneurysm showed statistically significant differences between the two groups ( $p$ value $=$ 0.024, 0.01, respectively).

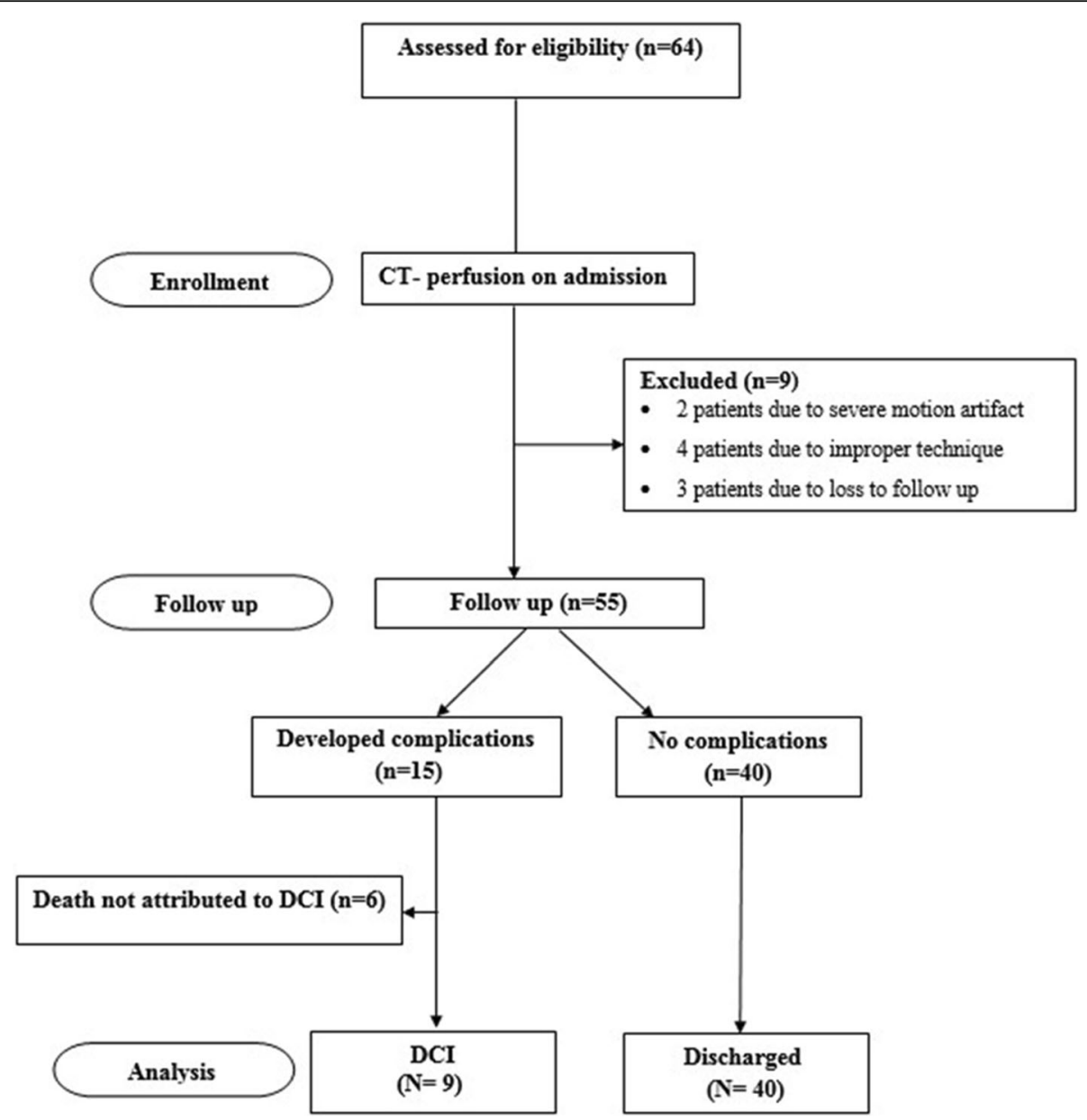

Fig. 1 Study flow chart. DCl, delayed cerebral ischemia 
Table 1 Baseline demographics in the DCl and Non-DCl groups

\begin{tabular}{|c|c|c|c|}
\hline Variable & $\mathrm{DCl}(N=9)$ & Non-DCI $(N=40)$ & $p$ value \\
\hline Age & $48.7(15.5)$ & $49.8(11.8)$ & 0.811 \\
\hline Age group & & & 1.0 \\
\hline$\geq 50$ years & $5(55.6 \%)$ & $20(50 \%)$ & \\
\hline$<50$ years & $4(44.4 \%)$ & $20(50 \%)$ & \\
\hline Gender (male\%) & $6(66.7 \%)$ & $21(52.5 \%)$ & 0.488 \\
\hline Hypertension & $2(22.2 \%)$ & $22(55 \%)$ & 0.138 \\
\hline Diabetes & $0(0 \%)$ & $7(17.5 \%)$ & 0.217 \\
\hline \multicolumn{4}{|l|}{ Hunt and Hess scale } \\
\hline 1 & $0(0 \%)$ & $24(60 \%)$ & $0.000^{\mathrm{a}}$ \\
\hline 2 & $0(0 \%)$ & $12(30 \%)$ & \\
\hline 3 & $5(55.6 \%)$ & $4(10 \%)$ & \\
\hline 4 & $2(22.2 \%)$ & $0(0 \%)$ & \\
\hline 5 & $2(22.2 \%)$ & $0(0 \%)$ & \\
\hline \multicolumn{4}{|l|}{ Hunt and Hess groups } \\
\hline$\geq 3$ & $9(100 \%)$ & $3(7.5 \%)$ & $0.000^{a}$ \\
\hline$<3$ & $0(0 \%)$ & 37 (92.5\%) & \\
\hline Modified Fisher score & & & $0.014^{a}$ \\
\hline 1 & $1(12.5 \%)$ & $24(60 \%)$ & \\
\hline 2 & $1(12.5 \%)$ & $7(17.5 \%)$ & \\
\hline 3 & $2(22.2 \%)$ & $2(5 \%)$ & \\
\hline 4 & $5(55.6 \%)$ & $7(17.5 \%)$ & \\
\hline \multicolumn{4}{|l|}{ Modified Fisher groups } \\
\hline$\geq 3$ & $7(77.8 \%)$ & $9(22.5 \%)$ & $0.003^{a}$ \\
\hline$<3$ & $2(22.2 \%)$ & $31(77.5 \%)$ & \\
\hline Intracerebral hematoma & $4(44.4 \%)$ & $6(15 \%)$ & 0.07 \\
\hline Site of SAH & & & $0.01^{\mathrm{a}}$ \\
\hline Anterior interhemispheric & $4(44.4 \%)$ & $7(17.4 \%)$ & \\
\hline Insular & $5(55.6 \%)$ & $6(15 \%)$ & \\
\hline Perimesencephalic & $0(0 \%)$ & $11(27.5 \%)$ & \\
\hline Global (non-specific) & $0(0 \%)$ & $16(40 \%)$ & \\
\hline Presence of aneurysm & $7(77.8 \%)$ & $14(35 \%)$ & $0.028^{a}$ \\
\hline Site of aneurysm & & & $0.01^{a}$ \\
\hline Anterior circulation & $7(77.8 \%)$ & $10(25 \%)$ & \\
\hline Posterior circulation & $0(0 \%)$ & $3(7.5 \%)$ & \\
\hline
\end{tabular}

$\mathrm{DCl}$ delayed cerebral ischemia

$N(\%)$ number and percentage

${ }^{\text {a }}$ Statistically significant

As regards the CTP parameters, only MTT showed a statistically significant difference, $p$ value 0.005 . None of the other absolute and relative parameters showed a statistically significant difference, shown in Table 2.

We found that neither age, gender, nor hypertension could predict DCI. Hunt and Hess scale had the most statistically significant predictive value $(p$ value $=0.002$, $\mathrm{OR}=8.766$ ). It is followed by Fisher scale that had comparable significant values $(p$ value $=0.006, \mathrm{OR}=2.511)$.
Table 2 Absolute and relative CTP values among patients with and without DCl

\begin{tabular}{llll}
\hline Variable & DCl & No-DCl & $p$ value \\
\hline CBF & $33.2(10.5$ & $42.4 \pm 16.0$ & 0.105 \\
CBV & $2.48 \pm 0.6$ & $2.37 \pm 0.8$ & 0.709 \\
MTT & $5.0 \pm 1.0$ & $3.9 \pm 1.0$ & $\mathbf{0 . 0 0 5 ^ { a }}$ \\
TTP & $17.6 \pm 4.9$ & $18.0 \pm 5.1$ & 0.801 \\
CBF difference & $19.4(17.8)$ & $9.0(7.9)$ & 0.187 \\
CBV difference & $-0.3(1.3)$ & $-0.2(0.5)$ & 0.826 \\
MTT difference & $0.68(0.8)$ & $0.4(0.8)$ & 0.147 \\
TTP difference & $-0.7(6.1)$ & $0.2(1.2)$ & 0.255 \\
CBF ratio & $0.8(0.4)$ & $0.9(0.1)$ & 0.067 \\
CBV ratio & $0.8(0.8)$ & $1.0(0.2)$ & 0.178 \\
MTT ratio & $1.2(0.3)$ & $1.1(0.3)$ & 0.245 \\
TTP ratio & $1.0(0.4)$ & $1.0(0.1)$ & 0.067 \\
\hline
\end{tabular}

Data expressed in mean (standard deviation)

$C B F$ cerebral blood flow, $C B V$ cerebral blood flow, $D C l$ delayed cerebral

ischemia, MTT mean transit time, TTP time to peak

${ }^{a}$ Statistically significant

The site of SAH did not exhibit predictive value ( $p$ value $=0.135)$. The presence of a cerebral aneurysm had a significant predictive value $(p$ value $=0.026$ and 0.03 , OR $=$ 9.5 and 6.5, respectively). Moreover, the anterior communicating artery aneurysm was a specific aneurysmal site related to DCI ( $p$ value $=0.026$, OR $=9.5)$. Of the perfusion parameters, only MTT had a significant predictive value $(p$ value $=0.024$, OR $=2.598)$ (Table 3$)$.

A ROC curve analysis including Hunt and Hess scale, Fisher-scale, the presence of an aneurysm, and prolonged MTT in a predictive model produced an area

Table 3 Logistic regression of predictors of DC

\begin{tabular}{llllll}
\hline & beta & $\begin{array}{l}p \\
\text { value }\end{array}$ & OR & \multicolumn{2}{c}{$95 \% \mathrm{Cl}$} \\
\cline { 5 - 7 } & & & & Lower & Upper \\
\hline AGE & 0.007 & 0.806 & 1.007 & 0.950 & 1.068 \\
Age group & 0.223 & 0.764 & 1.25 & 0.292 & 5.348 \\
Male gender & -0.593 & 0.553 & 0.553 & 0.121 & 2.523 \\
Hypertension & -1.453 & 0.092 & 0.234 & 0.043 & 1.267 \\
Hunt and Hess scale & 2.171 & $\mathbf{0 . 0 0 2 *}$ & 8.766 & 2.175 & 35.321 \\
Fisher scale & 0.921 & $\mathbf{0 . 0 0 6 *}$ & 2.511 & 1.299 & 4.851 \\
Site of SAH & -1.163 & 0.135 & 0.313 & 0.68 & 1.438 \\
Aneurysm & 1.872 & $\mathbf{0 . 0 3 *}$ & 6.5 & 1.187 & 35.598 \\
ACOM aneurysm & 2.251 & $\mathbf{0 . 0 2 6 *}$ & 9.5 & 1.304 & 69.191 \\
CBF & 0.047 & 0.159 & 1.048 & 0.982 & 1.118 \\
CBV & -0.054 & 0.116 & 0.947 & 0.885 & 1.014 \\
MTT & 0.955 & $\mathbf{0 . 0 2 4}$ & 2.598 & 1.137 & 5.937 \\
TTP & -0.019 & 0.796 & 0.981 & 0.849 & 1.133 \\
\hline ACOM ant
\end{tabular}

ACOM anterior communicating artery, $\mathrm{Cl}$ confidence interval, $\mathrm{OR}$ odds ratio, $\mathrm{SAH}$ subarachnoid hemorrhage

*Statistically significant difference $(p<0.05)$ 
under the curve of $0.894,0.794,0.714$, and 0.849 , respectively. The analysis demonstrated that Hunt and Hess scale $>2$ was the best potential predictor of DCI with sensitivity (88.9\%), specificity (90\%), PPV (66.7\%), NPV (97.3\%), and accuracy (78.89\%). Fisher scale $>2$ also predicted DCI with sensitivity (77.8\%), specificity (77.5\%), PPV (43.8\%), NPV (93.9\%), and accuracy (79.4\%). Presence of an aneurysm could predict DCI with sensitivity (77.8 \%), specificity (77.8\%), PPV (33.3\%), NPV (92.9\%), and accuracy (42.78\%). Anterior communicating artery aneurysm location as a predictor of DCI had low sensitivity (33.33\%), high specificity (95.0\%), PPV (60.0\%), NPV (86.4\%), and low accuracy (28.33\%). Prolonged MTT > $4.65 \mathrm{~s}$ could predict DCI with sensitivity (88.9\%), specificity (72.5\%), PPV (42.1\%), NPV (96.7\%), and accuracy (61.39\%) (Table 4) and (Fig. 2).

We found that the early qualitative CTP could not discriminate minor perfusion deficits (Fig. 3), while late CTP performed closely after clinical deterioration could clearly depict perfusion deficit (Fig. 4). We found that the presence of early vasospasm does not inevitably lead to DCI (Fig. 5). We found that most of the patients recovered from SAH without developing vasospasm or DCI (Fig. 6).

\section{Discussion}

Up to our knowledge, this is the first study investigating this issue in the Middle East population. Also, it is the first prospective study investigating the predictive value of many clinical and radiological parameters, including CTP, that may have a role in the development of DCI.

We found that age was not a great risk factor for DCI and the occurrence of DCI was not affected by age group. This finding agrees with those of Gross et al. [18] and Zaidat et al. [19] who showed that age was not a predictor of DCI. These results differ from that of Said et al. [20] who revealed a better outcome in the younger age group $(p=0.007)$. These contradictory results may be partly explained by the differences in patients' demographics.

We also found that a patient's gender was not a significant predictor of DCI. Also, a study conducted by Gross et al. [18] found the same results.
Hypertension also had no significant predictive value of DCI (OR 0.234, $p=0.09$ ), in agreement with a previous study [21]. This can be explained as all patients were receiving nimodipine, a calcium channel blocker, preventing vasospasm by its vasodilator effect and subsequently controlling the blood pressure [22].

The most important clinically relevant finding in our study was Hunt and Hess scale where high scales were significantly associated with a higher likelihood of DCI (OR 8.766, $p=0.002$ ) with sensitivity $88.9 \%$ and specificity $90 \%$. We found that Hunt and Hess scale was the most sensitive and specific predictor among all studied predictors $(A U C=0.849)$. This study supports evidence from the previously published studies [23, 24]. High Hunt and Hess scales are clearly associated with poor survival rates.

Another important finding was the value of Fisher scale as a predictor of DCI. We found that high Fisher scales were significantly associated with a higher likelihood of DCI (OR 2.511, $p=0.006$ ) with a sensitivity of $77.8 \%$ and specificity $77.5 \%$. In accordance with the present results, previous studies demonstrated that high Fisher scale was associated with a greater risk of DCI $[23,24]$. The more amount of subarachnoid blood adds more irritative injury to the brain.

Previous studies evaluating the risk of a cerebral aneurysm in developing vasospasm and DCI observed higher likelihood in aneurysmal SAH versus nonaneurysmal SAH (e.g., perimesencephalic SAH) (OR $3.79, p=0.009$ ) [25]. Our results support this evidence from previous studies as we found that the presence of a cerebral aneurysm was associated with a higher probability of DCI (OR 6.5, $p=0.03$ ), in accordance with what previously published [26]. Moreover, we found that ACOM aneurysm had an additive risk of DCI (OR 9.5, $p$ $=0.026$ ) having the worst disease course compared to other aneurysmal locations. This result agrees with the previous conclusion described by Abla et al. [27].

Many studies investigated the CTP parameters in predicting and diagnosing DCI. Some researchers used the absolute values of CTP and others used the relative values which are the differences and ratios between both cerebral hemispheres. The predictive role of CTP is still up till now controversial [28]. In this study, we measured

Table 4 Sensitivity, specificity, PPV, PPV, accuracy, and AUC of the potential predictors

\begin{tabular}{llllllll}
\hline & Cut-off value & Sensitivity & Specificity & PPV & NPV & Accuracy & AUC \\
\hline Hunt & $>2$ & $88.9 \%$ & $90 \%$ & $66.7 \%$ & $97.3 \%$ & $78.89 \%$ & 0.894 \\
Fisher & $>2$ & $77.8 \%$ & $77.5 \%$ & $43.8 \%$ & $93.9 \%$ & $79.40 \%$ & 0.794 \\
Aneurysm & $>$ & $77.8 \%$ & $65 \%$ & $33.3 \%$ & $92.9 \%$ & $42.78 \%$ & 0.714 \\
ACOM aneurysm & $>0$ & $33.33 \%$ & $95 \%$ & $60 \%$ & $86.4 \%$ & $28.33 \%$ & 0.642 \\
MTT & 4.65 & $71.40 \%$ & $87.50 \%$ & $42.1 \%$ & $96.7 \%$ & $61.39 \%$ & 0.849 \\
\hline
\end{tabular}

ACOM anterior communicating artery, PPV positive predictive value, NPV negative predictive value, AUC area under the curve 

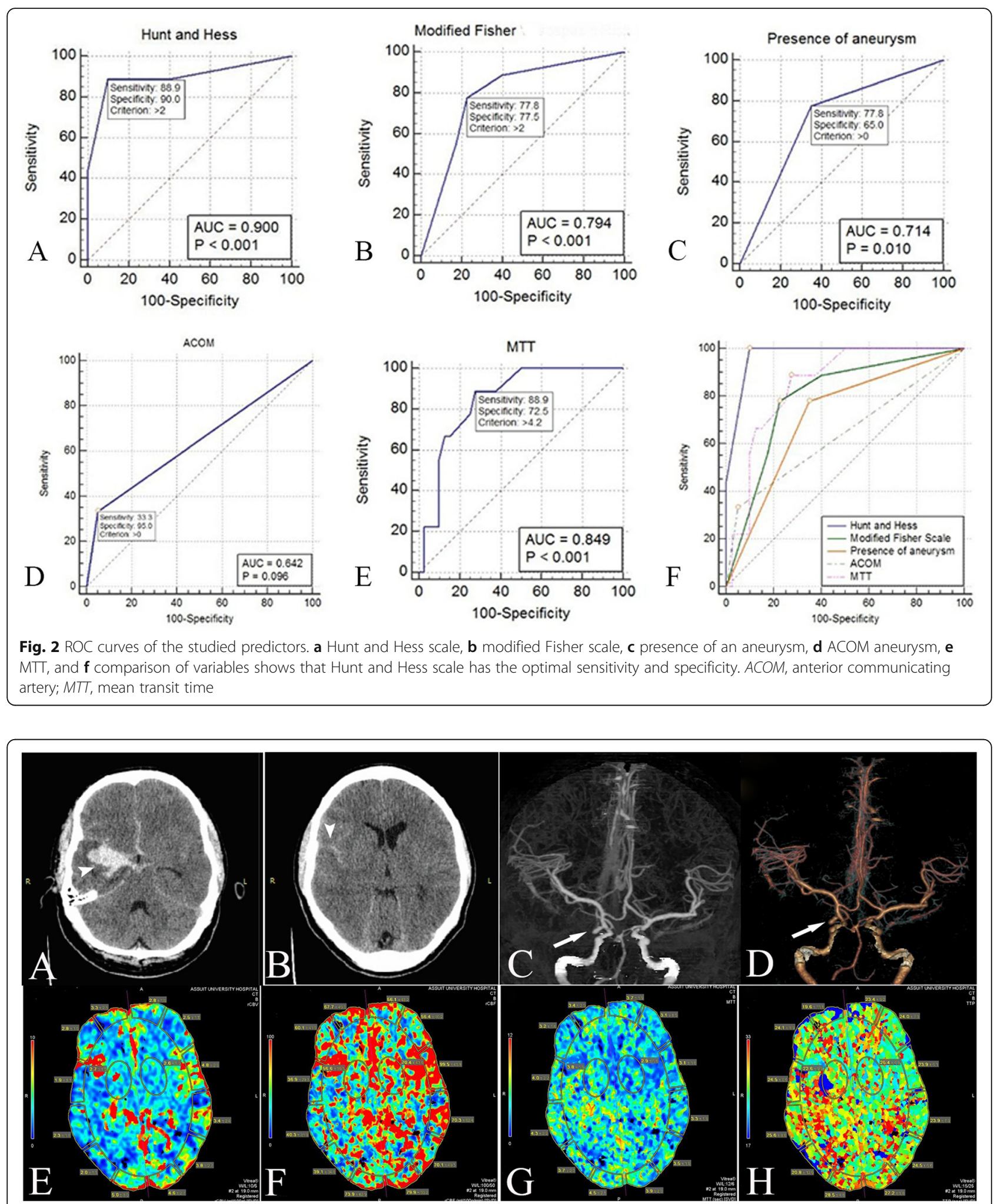

Fig. 3 A 36-year-old male patient presented with severe headache, neck stiffness, and mild left-sided hemiparesis. Non-contrast CT (a, b) shows right temporal hematoma and SAH within the right Sylvian and anterior interhemispheric fissures (asterisks in $\mathbf{a}$ and $\mathbf{b}$ ). MIP (c) and volume rendering (d) images of CTA show a saccular aneurysm at the right internal carotid artery; posterior communicating segment (arrowheads in $\mathbf{c}$ and $\mathbf{d})$. No vasospasm. CT perfusion maps show subtle perfusion deficit on the right-side following territory of right MCA; CBV (e), CBF (f), MTT $(\mathbf{g})$, and $T \mathrm{P}(\mathbf{h})$ 


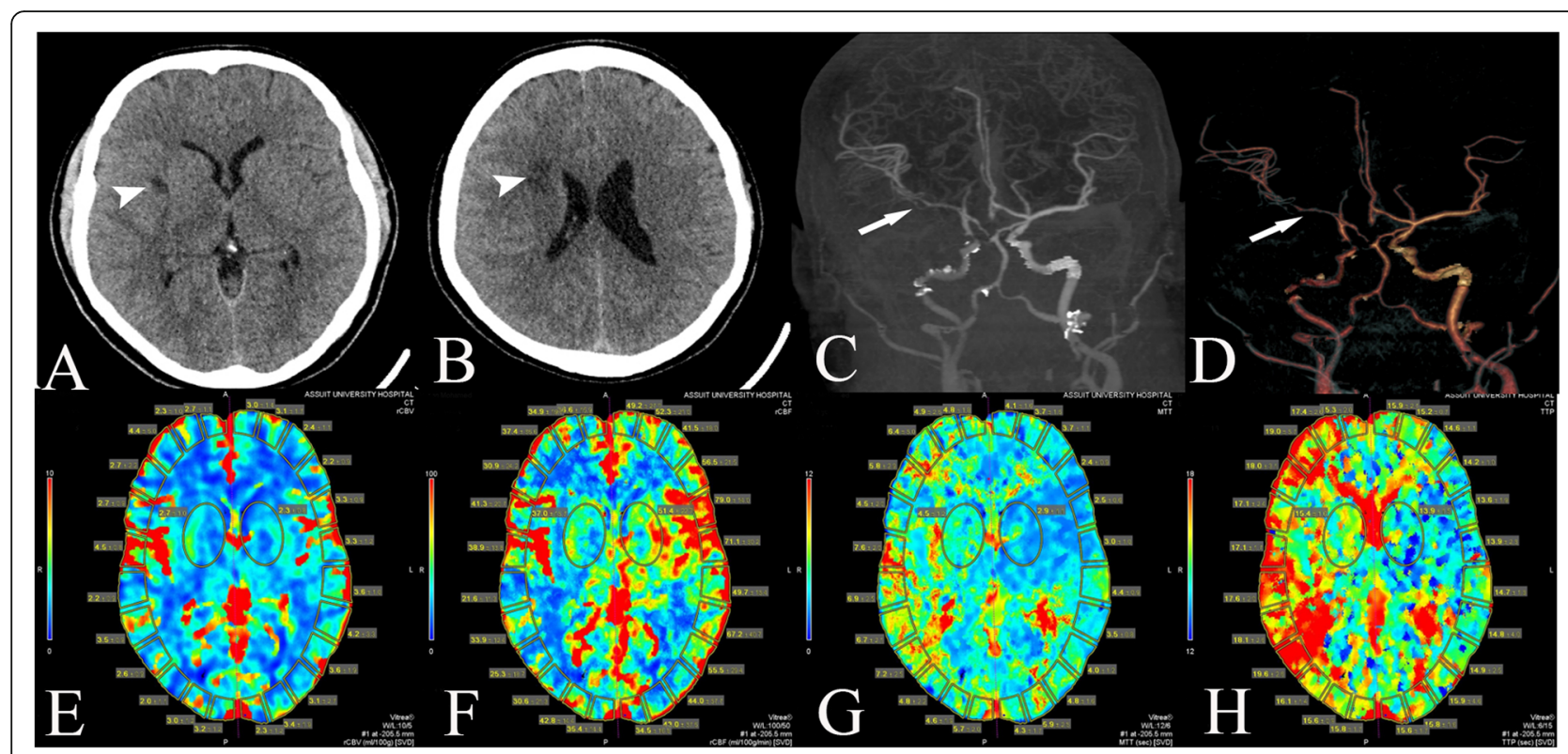

Fig. 4 On follow-up after 10 days, the patient developed left-sided hemiparesis and confusion. Non-contrast CT (a, b) shows multiple infarcts involving the right lentiform nucleus (asterisk in $\mathbf{a}$ ) and right frontal periventricular white matter (asterisk in b). MIP (c) and volume rendering (d) images of CTA show severe vasospasm of the right MCA segments M1 and M2 (arrows in $\mathbf{c}$ and $\mathbf{d}$ ) as well as the right ACA segment A1. CT perfusion maps show significant perfusion deficit at the right side; reduced CBV (e), reduced CBF $(\mathbf{f})$, prolonged MTT (g), and prolonged TTP (h)

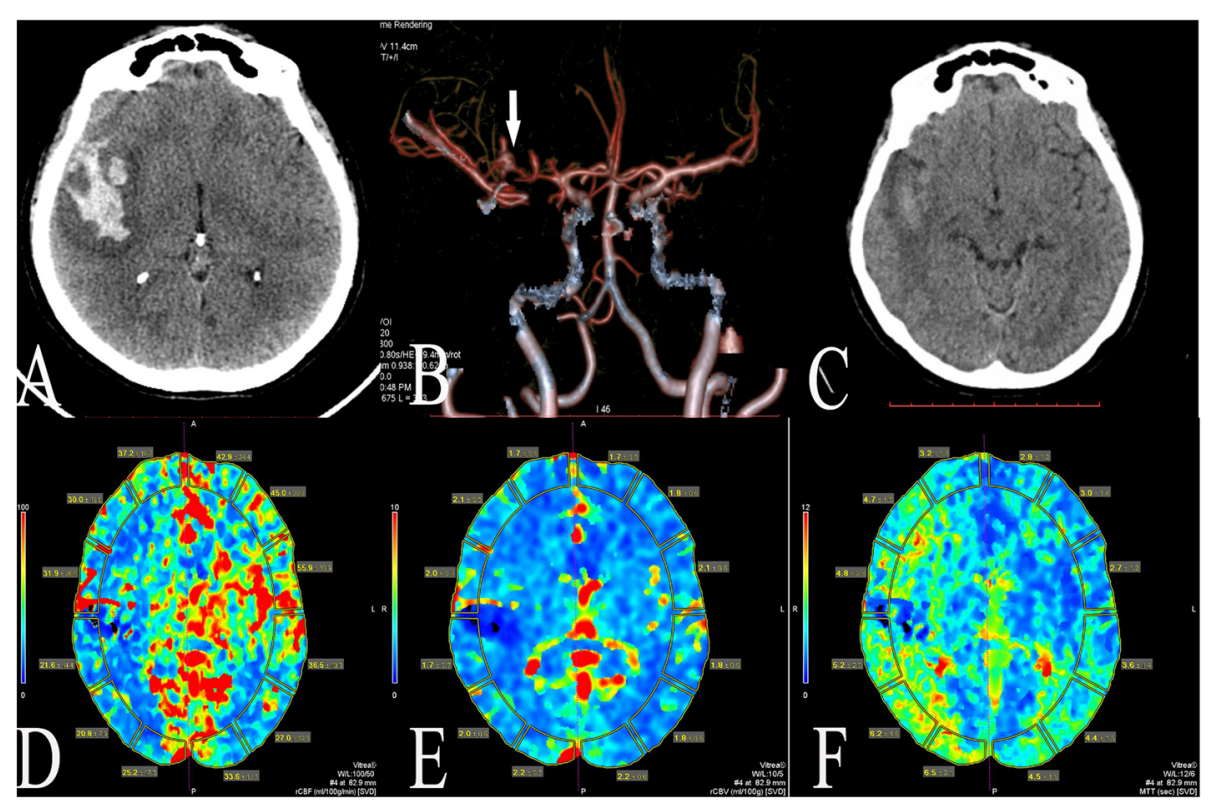

Fig. 5 A 45-year-old female patient presented with severe headache and neck stiffness of 1-day duration with no motor deficit. On admission non-contrast CT (a) shows right temporal hematoma with surrounding edema and right Sylvian fissure SAH. Volume rendering image (b) of CTA done 1 day after SAH shows a right MCA saccular aneurysm (arrow) having multiple off branches. Moderate vasospasm of the right MCA M1 and M2 segments. Multiple collaterals are evident. Follow-up CT on discharge after 15 days (c) shows reduction of size and density of the hematoma, i.e., clot retraction, with no infarcts. CT perfusion maps (d-f) done 1 day after SAH revealed mild perfusion deficit on the right side; reduced CBF (d), maintained CBV (e), and prolonged MTT (e). The maintained CBV despite vasospasm is due to collaterals and autoregulation 


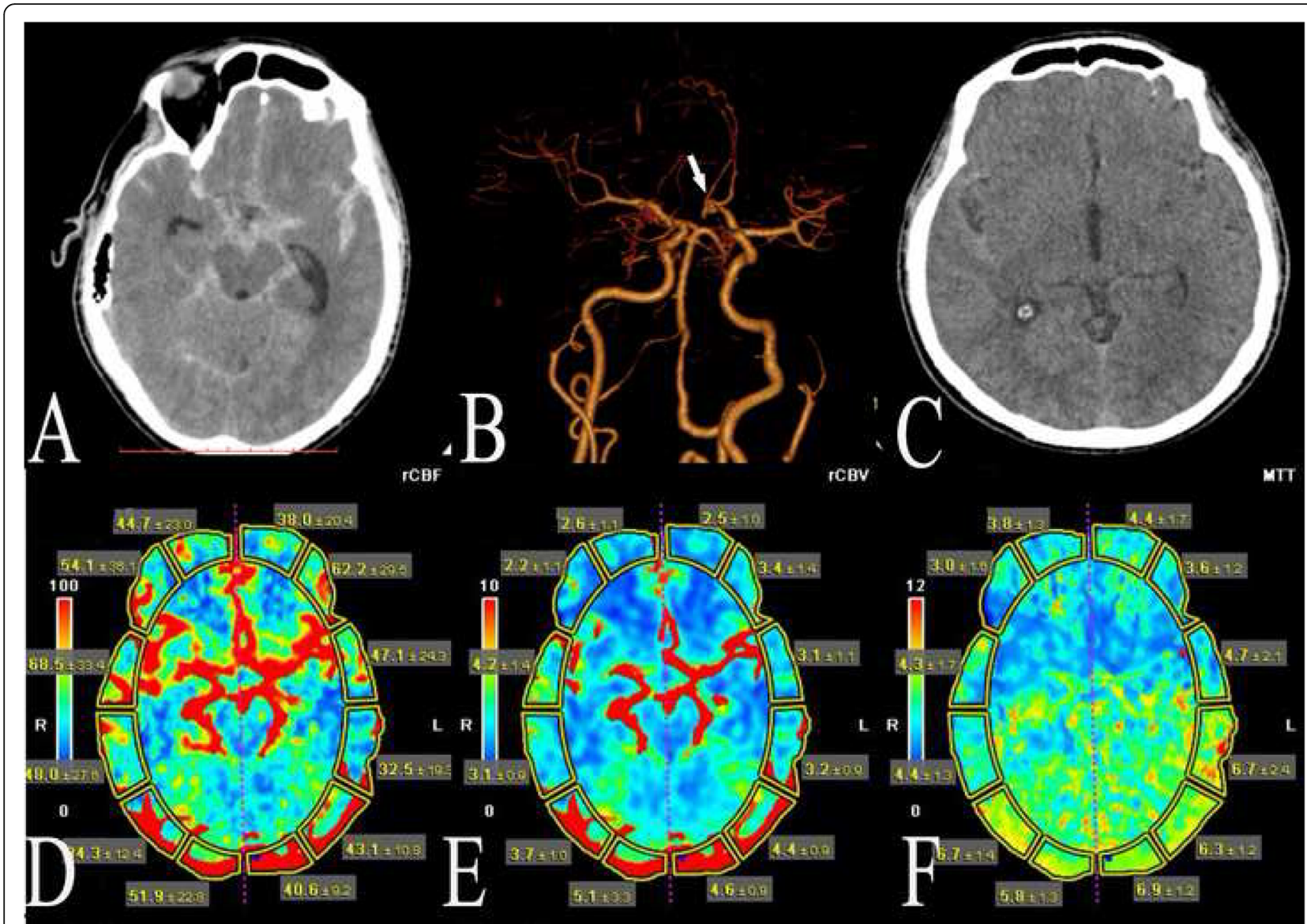

Fig. 6 A 48-year-old male patient presented with mild headache and neck stiffness of 1-day duration with no motor deficit. On admission noncontrast CT (a) shows global SAH mainly in the anterior interhemispheric, suprasellar, and left Sylvian cisterns. Volume rendering image (b) of CTA done 1 day after SAH shows an ACOM saccular aneurysm is seen (arrow) and absent right A1 segment of right ACA. Follow-up CT on discharge after 16 days (c) near-total resolution of SAH with no infarcts. CT perfusion maps ( $\mathbf{d}-\mathbf{f}$ ) done 1 day after SAH revealed normal perfusion parameters $\mathrm{CBF}$ (d), CBV (e), and MTT (e)

CBF, CBV, MTT, and TTP in the area of least perfusion. Also, we calculated the relative CTP values.

As regards the absolute CTP values, we found that prolonged MTT was the only parameter that had a statistically significant difference between the DCI group and non-DCI group. We found that MTT prolongation is the most sensitive perfusion parameter to detect early ischemic events before becoming clinically evident. MTT threshold > $4.65 \mathrm{~s}$ can predict DCI with sensitivity (88.9\%), specificity (72.5\%), PPV (42.1\%), NPN (96.7\%), and accuracy (61.39\%). None of the other parameters showed any statistically significant difference between the two groups. CBF was even lower in those who developed DCI; however, its $p$ value was not statistically significant. A previous study investigated the absolute CTP values in predicting DCI found near our results as regards the MTT with a much higher threshold of $5.9 \mathrm{~s}$ and found a sensitivity of $50 \%$ and a specificity of $95 \%$ [29]. Also, many other reports support the role of MTT in predicting DCI [30, 31].

As regards the relative CTP values obtained in our study, we found DCI was not significantly related to such relative perfusion values. None of these values could exhibit any statistically significant difference between the two groups including differences and ratios, with subsequent low sensitivity and specificity when entering their values in ROC curve analysis. A study made by van der Schaaf et al. [13] investigated the relative CTP values and found that $\mathrm{CBF}$ and CBV ratios and MTT and TTP differences had predictive thresholds with moderate sensitivities and specificities. This discrepancy in results can be explained by the different methods of perfusion measurement. Also, many patients have bilateral and diffuse ischemia after SAH and the interhemispheric differences of measurements might be not significant. Therefore, the relative CTP parameters had worse diagnostic properties [32]. 
A strength of this study is that the usage of Vitrea workstation that has the advantage of a sharp definition of the arterial input function (AIF) via crosshair avoiding the partial volume effect of region of interest (ROI) ellipse. Another strong point is the sharp definition of DCI and the exclusion of patients with other causes of deterioration such as rebleeding.

Our study had several potential limitations. Given that the number of patients with SAH in our study is still small, especially for subgroup analyses, further research of a larger sample size is needed. However, such sample size was due to the limited period of the study and a relatively lower incidence of SAH in our region. Also, this study includes aneurysmal and non-aneurysmal SAH patients that may play a confounding role in the results.

\section{Conclusion}

Hunt and Hess scale has the optimal sensitivity and specificity to distinguish between patients who developed DCI and clinically stable patients. Also, Fisher scale and MTT are sensitive and specific predictors of DCI. The presence and site of an aneurysm also raise the risk of DCI.

\begin{abstract}
Abbreviations
ACA: Anterior cerebral artery; ACOM: Anterior communicating artery; CBF: Cerebral blood flow; CBV: Cerebral blood volume; CTA: Computed tomography angiography; CTP: Computed tomography perfusion; DCl: Delayed cerebral ischemia; DWI: Diffusion-weighted imaging; FLAIR: Fluid attenuation recovery; MCA: Middle cerebral artery; MIP: Maximum intensity projection; MRI: Magnetic resonance imaging; MTT: Mean transit time; NCT: Number of clinical trials; OR: Odds ratio; PCA: Posterior cerebral artery; SAH: Subarachnoid hemorrhage; TTP: Time to peak
\end{abstract}

\section{Acknowledgements}

The authors thank all the study participants for their patience and support.

\section{Authors' contributions}

A. A. H. and H. A. S. suggest and develop the research idea, reviewing literature.

A. A. H., M. A. A., H. A. S., and M. A. were responsible for data collection and analysis, perform statistical analysis, write and revise the manuscript, prepare cases, and perform required measurements, prepare figures, and tables. - M. A. and H. A.Y. were responsible for reporting the cases of CT perfusion and refer to us a result to compare it with our radiological results.

- All authors have a major contribution in preparing and editing the manuscript.

- All authors read and approved the final manuscript.

\section{Funding}

This study was financially supported by Grant office of the Faculty of Medicine, Assiut University.

\section{Availability of data and materials}

The data that support the findings of this study are available from Radiology Department, Assiut University, but there are restrictions apply to availability of data, which used under license for this study, and so weren't publicly available. Data were available from authors upon request with permission of head of Radiology Department, Assiut University.

\section{Ethics approval and consent to participate}

This study had approval from Egypt, Assiut University, Faculty of Medicine Research Ethics Committee. All patients participated in this study signed an informed written consent for participation.

\section{Ethical consideration}

1. Risk Benefit assessment for all patients were indicated for the examination during this study.

2. Confidentiality: All patients' data were confidentially kept.

3. The research done by scientifically qualified and trained personnel only.

4. The procedures included in this study had been already used in hospital and centers in and outside Egypt.

Consent for publication

A consent to publish was obtained.

\section{Competing interests}

The authors declare that they have no competing interests.

\section{Author details}

${ }^{1}$ Department of Radiology, Faculty of Medicine, Assiut University, Assiut, Egypt. ${ }^{2}$ Department of Neurology, Faculty of Medicine, Assiut University, Assiut, Egypt.

Received: 10 February 2020 Accepted: 15 April 2020

Published online: 21 April 2020

\section{References}

1. Kooijman E, Nijboer CH, Van Velthoven CTJ, Mol W, Dijkhuizen RM, Kesecioglu J et al (2014) Long-term functional consequences and ongoing cerebral inflammation after subarachnoid hemorrhage in the rat. PLoS One 9:e90584

2. Grasso G (2015) Vasospasm in aneurysmal subarachnoid hemorrhage: An evolving knowledge. World Neurosurg 83:258-259

3. Lord A, Fernandez L, Schmidt J, Mayer S, Claassen J, Lee K et al (2011) Effect of rebleeding on the course and incidence of vasospasm after subarachnoid hemorrhage. Neurology 78(1):31-37

4. Li J, Su L, Ma J, Kang P, Ma L, Ma L (2017) Endovascular coiling versus microsurgical clipping for patients with ruptured very small intracranial aneurysms: management strategies and clinical outcomes of 162 Cases. World Neurosurg 99:763-769

5. D'Souza S (2015) Aneurysmal subarachnoid hemorrhage. J Neurosurg Anesthesiol 27:222-240

6. Budohoski KP, Czosnyka M, Smielewski P, Kasprowicz M, Helmy A, Bulters D et al (2012) Impairment of cerebral autoregulation predicts delayed cerebral ischemia after subarachnoid hemorrhage: A prospective observational study. Stroke 43:3230-3237

7. Roy B, McCullough L, Dhar R, Grady J, Wang Y, Brown R (2017) Comparison of initial vasopressors used for delayed cerebral ischemia after aneurysmal subarachnoid hemorrhage. Cerebrovascular Dis 43(5-6):266-271

8. Killeen RP, Mushlin Al, Johnson CE, Comunale JP, Tsiouris AJ, Delaney H et al (2011) Comparison of CT perfusion and digital subtraction angiography in the evaluation of delayed cerebral ischemia. Acad Radiol 18: 1094-1100

9. Mortimer AM, Steinfort B, Faulder K, Harrington T (2016) Delayed infarction following aneurysmal subarachnoid hemorrhage: can the role of severe angiographic vasospasm really be dismissed? J Neurointerv Surg 8:802-807

10. Bacigaluppi S, Zona G, Secci F, Spena G, Mavilio N, Brusa G et al (2015) Diagnosis of cerebral vasospasm and risk of delayed cerebral ischemia related to aneurysmal subarachnoid haemorrhage: an overview of available tools. Neurosurg Rev 38:603-618

11. Rodriguez-Régent C, Hafsa M, Turc G, Ben Hassen W, Edjlali M, Sermet A et al (2016) Early quantitative CT perfusion parameters variation for prediction of delayed cerebral ischemia following aneurysmal subarachnoid hemorrhage. Eur Radiol 26:2956-2963

12. Kozak N, Bereczki D, Szabo S (2016) Predictors of symptomatic vasospasm after subarachnoid hemorrhage: a single center study of 457 consecutive cases. Turk Neurosurg 26:545-549

13. Dai DW, Zhao WY, Zhang YW, Yang ZG, Li Q, Xu B et al (2013) Role of CT perfusion imaging in evaluating the effects of multiple burr hole surgery on adult ischemic Moyamoya disease. Neuroradiology 55:1431-1438

14. Bae KT (2010) Intravenous contrast medium administration and scan timing at CT: considerations and approaches. Radiology 256:32-61 
15. Bao DZ, Bao HY, Yao LZ, Pan YG, Zhu XR, Yang XS et al (2013) 64-Slice spiral CT perfusion combined with vascular imaging of acute ischemic stroke for assessment of infarct core and penumbra. Exp Ther Med 6:133-139

16. Okorie C, Ogbole G, Owolabi M, Ogun O, Adeyinka A, Ogunniyi A (2015) Role of diffusion-weighted imaging in acute stroke management using lowfield magnetic resonance imaging in resource-limited settings. West African J Radiol 22:61

17. Bivard A, Levi C, Spratt N, Parsons M (2013) Perfusion CT in acute stroke: a comprehensive analysis of infarct and penumbra. Radiology 267:543-550

18. Gross BA, Rosalind Lai PM, Frerichs KU, Du R (2014) Treatment modality and vasospasm after aneurysmal subarachnoid hemorrhage. World Neurosurg 82:E725-E730

19. Zaidat OO, Ionita CC, Hussain SI, Alexander MJ, Friedman AH, Graffagnino C (2009) Impact of ruptured cerebral aneurysm coiling and clipping on the incidence of cerebral vasospasm and clinical outcome. J Neuroimaging 19: 144-149

20. Said AHM, El-Ghandour NMF (2015) Outcome of aneurismal subarachnoid hemorrhage: how far is vasospasm involved? - Retrospective study. Egypt J Radiol Nucl Med 46:111-116

21. Tewari M, Aggarwal A, Mathuriya S, Gupta V (2015) The outcome after aneurysmal sub arachnoid hemorrhage: a study of various factors. Ann Neurosci 22:78-80

22. Li Y, Fang W, Tao L, Li M, Yang Y, Gao Y et al (2015) Efficacy and safety of intravenous nimodipine administration for treatment of hypertension in patients with intracerebral hemorrhage. Neuropsychiatr Dis Treat 11:1231-1238

23. Ko S-B, Choi HA, Carpenter AM, Helbok R, Schmidt JM, Badjatia N et al (2011) Quantitative analysis of hemorrhage volume for predicting delayed cerebral ischemia after subarachnoid hemorrhage. Stroke 42(3):669-674

24. Yin L, Ma CY, Li ZK, Wang DD, Bai CM (2011) Predictors analysis of symptomatic cerebral vasospasm after subarachnoid hemorrhage. Acta Neurochir Suppl 110:175-178

25. Gross BA, Lin N, Frerichs KU, Du R (2012) Vasospasm after spontaneous angiographically negative subarachnoid hemorrhage. Acta Neurochir (Wien) 154:1127-1133

26. Lago A, López-Cuevas R, Tembl Jl, Fortea G, Górriz D, Aparici F et al (2016) Short- and long-term outcomes in non-aneurysmal non-perimesencephalic subarachnoid hemorrhage. Neurol Res 38:692-697

27. Abla AA, Wilson DA, Williamson RW, Nakaji P, McDougall CG, Zabramski JM et al (2014) The relationship between ruptured aneurysm location, subarachnoid hemorrhage clot thickness, and incidence of radiographic or symptomatic vasospasm in patients enrolled in a prospective randomized controlled trial. J Neurosurg 120:391-397

28. Rodriguez-Régent C, Hafsa M, Turc G, Ben Hassen W, Edjlali M, Sermet A et al (2015) Early quantitative CT perfusion parameters variation for prediction of delayed cerebral ischemia following aneurysmal subarachnoid hemorrhage. European Radiology. 26(9):2956-2963

29. Lagares A, Cicuendez M, Ramos A, Salvador E, Alén J, Kaen A et al (2012) Acute perfusion changes after spontaneous SAH: a perfusion CT study. Acta Neurochirurgica 154(3):405-412

30. Etminan N, Beseoglu K, Heiroth HJ, Turowski B, Steiger HJ, Hänggi D (2013) Early perfusion computerized tomography imaging as a radiographic surrogate for delayed cerebral ischemia and functional outcome after subarachnoid hemorrhage. Stroke 44:1260-1266

31. Sanelli PC, Anumula N, Johnson CE, Comunale JP, Tsiouris AJ, Riina H et al (2013) Evaluating CT perfusion using outcome measures of delayed cerebral ischemia in aneurysmal subarachnoid hemorrhage. Am J Neuroradiol 34: 292-298

32. You C, Zhang H, Liu Y, Ma J, Sun H, Wang K (2013) Accuracy of computed tomography perfusion in detecting delayed cerebral ischemia following aneurysmal subarachnoid hemorrhage: A meta-analysis. Neurol India 61:507

\section{Publisher's Note}

Springer Nature remains neutral with regard to jurisdictional claims in published maps and institutional affiliations.

\section{Submit your manuscript to a SpringerOpen ${ }^{\circ}$ journal and benefit from:}

- Convenient online submission

- Rigorous peer review

- Open access: articles freely available online

- High visibility within the field

- Retaining the copyright to your article

Submit your next manuscript at $\boldsymbol{\nabla}$ springeropen.com 